\title{
Molecular markers guide diagnosis and treatment in Philadelphia chromosome-negative myeloproliferative disorders (Review)
}

\author{
ANA MARIA VLADAREANU ${ }^{1}$, CARSTEN MÜLLER-TIDOW ${ }^{2}$, HORIA BUMBEA $^{1}$ and SINZIANA RADESI $^{1}$ \\ ${ }^{1}$ Emergency University Hospital Bucharest, 'Carol Davila' University of Medicine and Pharmacy, Romania; \\ ${ }^{2}$ Department of Internal Medicine, Hematology and Oncology, University of Münster, Germany
}

Received October 13, 2009; Accepted November 30, 2009

DOI: 10.3892/or_00000674

\begin{abstract}
The Philadelphia negative chronic myeloproliferative neoplasms are hematological disorders with several diagnostic challenges. Due to recent molecular findings, the WHO classification of Tumors of Hematopoietic and Lymphoid Tissue 2008 reorganized the field of chronic myeloproliferative diseases. Thus, specific molecular markers provide important information for current diagnostic strategies. This review highlights the important diagnostic tools in classical and atypical myeloproliferative neoplasms mainly the JAK2V617F mutation, the Mpl receptor, Polycythemia rubra vera 1 (PRV1), platelet-derived growthfactor receptor $\alpha$ (PDGFRA), platelet-derived growth-factor receptor $B$ (PDGFRB), fibroblast growth-factor receptor 1 (FGFR1) and c-kit tyrosine kinase. A description of the origin, clinical correlations and role in diagnosis and therapy is provided for each of these molecular markers.
\end{abstract}

\section{Contents}

1. Chronic myeloproliferative disorders

2. The role of molecular markers in myeloproliferative diseases

3. Conclusions

\section{Chronic myeloproliferative disorders}

Chronic myeloproliferative disorders (CMPD) are a group of heterogeneous diseases with distinct prognostic implications. Until recently, diagnosis and classification were mostly based on clinical presentation, blood cell counts and bone marrow cytology. Diagnosis was difficult and it was often based on exclusion of a multitude of secondary alterations. In many cases, the distinction proved impossible, e.g., between

Correspondence to: Professor Ana-Maria Vladareanu, Department of Hematology, Emergency Universitary Hospital Bucharest, 169 Splaiul Independentei, 050098 Bucharest, Romania

E-mail: anamariavladareanu@yahoo.com

Key words: chronic myeloproliferative neoplasms, molecular markers, JAK2 mutation reactive platelet elevation and essential thrombocytosis. Recently, important discoveries were made with regard to specific mutations in CMPD. These basic research findings were rapidly translated into clinical practice. The most recent World Health Organization (WHO) classification of hematopoietic tumors, published in 2008 , brings changes by replacing the term CMPD to myeloproliferative neoplasms (MPN) and by organizing the classical and the atypical myeloproliferative syndromes in new subcategories based on their molecular pathogenesis (1).

This diagnosis-oriented molecular approach was first proposed as semi-molecular classification (see Table I) and implemented by the WHO in 2008 (Table II).

Despite their clinical and phenotypic heterogeneity, CMPD/ MPN share similar pathogenic mechanisms that involve the disturbance of tyrosine-kinase receptors or cytokine receptors (2) (Table III).

Therefore, protein molecular markers are associated with different subtypes of myeloproliferative syndromes, with implications in the pathogenesis (Table IV).

Chronic myeloid leukemia (CML) today serves as a paradigm in terms of molecular diagnosis, molecular monitoring and targeted therapy. Elucidation of the pathogenetic mechanisms of CML started in 1973 by the identification of the typical $\mathrm{t}(9 ; 22)$ mutation. Much later, the translocated genes were identified and the molecular mechanisms of CML were studied in detail $(3-5)$. The $t(9,22)$ ( $q 34 ; q 11)$ translocation, (Philadelphia chromosome-Ph1) generates the BCR-ABL chimeric fusion protein. BCR-ABL dyslocalizes the abl tyrosine kinase activity into the cytoplasm. Subsequently activation of multiple cytoplasmic signaling pathways occurs. Main downstream signaling pathways are the Ras/Mitogenactivated protein kinase (Ras/MAPK), phosphatidyl inositol-3 kinase/AKT (PI3K/AKT), and signal transducer and activator of transcription (STAT) pathways. These pathways are implicated in mitogenic signaling and enhancement of cellular survival. The development and the world wide use of abl tyrosine kinase inhibitors have dramatically improved CML patients' prognosis.

The WHO reformation of the $\mathrm{pH}$-negative MPN classification emphasizes the importance of the recently discovered genetic mutations (Table I) in the diagnosis of these diseases. The following molecular anomalies imposed themselves as hallmarks in the pathogenesis of these myeloproliferative syndromes: i) acquired point mutations of the gene encoding 
Table I. Semi-molecular classification of chronic myeloproliferative diseases. ${ }^{\mathrm{a}}$

\begin{tabular}{lll}
\hline Main class & \multicolumn{1}{c}{ Clinical pathogenic entities } & \multicolumn{1}{c}{ Molecular traits } \\
\hline Classic & BCR-ABL positive & Chronic myeloid leukemia $(\mathrm{CML})$ \\
MPS & BCR-ABL negative & Polycythemia vera (PV) $\left(\sim 100 \% J A K 2 V 617 F^{+}\right)$ \\
& & Essential thrombocythemia $(\mathrm{ET})\left(\sim 50 \% J A K 2 V 617 F^{+}\right)$ \\
& & Myeloid metaplasia with myelofibrosis $(\mathrm{MMM})$ \\
& & $\left(\sim 50 \% J A K 2 V 617 F^{+}\right)$
\end{tabular}

Atypical/ Chronic myelomonocytic leukemia (CMML)

borderline Juvenile myelomonocytic leukemia (JMMC):

MPS frequent mutations PTP11, NF1 and RAS

Chronic neutrophilic leukemia (CNL) $(\sim 20 \%$

$J A K 2 V 617 F^{+}$)

Chronic eosinophilic leukemia (CEL)/myeloproliferative diseases associated with eosinophilia

PDGFRA gene rearrangements (ex: FIP1L1-PDGFRA)

PDGFRB gene rearrangements (ex: TEL/ETV6-PDGFRA)

FGFR1 gene rearrangements (ex: ZNF198/FIM/RAMP-FGFR1; 8p11 myeloproliferative syndrome)

Hypereosinophilic syndrome

CEL uncharacterized molecular

Chronic basophilic leukemia

Systemic mastocytosis (SM)

PDGFRA gene rearrangements (ex: FIP1L1-PDGFRA)

KIT mutations (ex: KITD816V)

Unclassified MPS ( 20\% JAK2V617F+

SM uncharacterized molecular

mixed/overlapped MDS/MPS (including RARS-T)

CML-like syndromes, BCR-ABL negative

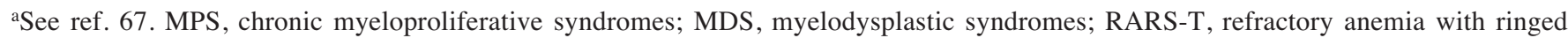
sideroblasts associated with thrombocytosis and megakaryocytic anomalies similar those found in MPS.

Table II. Chronic myeloproliferative neoplasms classification according to the novel WHO criteria. ${ }^{\text {a }}$

\begin{tabular}{ll}
\hline Main category & \multicolumn{1}{c}{ Subtypes } \\
\hline Myeloproliferative neoplasms & Chronic myelogenous leukaemia BCR-ABL1 positive \\
& Chronic neutrophilic leukaemia \\
& Polycythaemia vera \\
& Primary myelofibrosis \\
& Essential thrombocythaemia \\
& Chronic eosinophilic leukaemia, NOS \\
& Mastocytosis \\
& Cutaneous mastocytosis \\
& Systemic mastocytosis \\
& Mast cell leukaemia \\
& Mast cell sarcoma \\
& Extracutaneous mastocytoma \\
Myeloid and lymphoid neoplasms & Myeloproliferative neoplasm, unclassifiable \\
with eosinophilia and abnormalities of & Myeloid and lymphoid neoplasms with PDGFRA rearrangements \\
PDGFRA, PDGFRB and FDGFR-1 & Myeloid and lymphoid neoplasms with PDGFRB rearrangements \\
Myelodysplastic/myeloproliferative & Myeloid and lymphoid neoplasms with FGFR-1 rearrangements \\
neoplasms & Chronic myelomonocytic leukaemia \\
& Atypical chronic myeloid leukaemia BCR-ABL1 negative \\
& Juvenile myelomonocytic leukaemia \\
& Myelodysplastic/myeloproliferative neoplasms, unclassifiable \\
& Refractory anemia with ring sideroblasts associated with marked thrombocytosis \\
&
\end{tabular}

NOS, not otherwise specified. ${ }^{a}$ See ref. $1 .{ }^{b}$ Provisional entity, supplementary data required. 
Table III. Genetic lesions associated with myeloproliferative syndromes.

\section{Genetic defect}

$B C R-A B L$

$J A K 2 V 617 F$

\section{$M P L \mathrm{~W} 515 \mathrm{~K} / \mathrm{L}$}

KIT gene mutations

FIPIL1-PDGFRA

PDGFRB fusion gene

FGFR fusion gene

Trisomy 9

Trisomy 8

Trisomy $1 \mathrm{q}$

20q deletion

$5 q$ and $7 q$ deletions

$13 \mathrm{q}$ deletion
Associated disease

Dissemination in population $(\%)$
Chronic myeloid leukemia

Polycythemia vera

Essential thrombocythemia

Myeloid metaplasia with myelofibrosis

Other myeloid lineage affections

Essential thrombocythemia

Myeloid metaplasia with myelofibrosis

Systemic mastocytosis

Eosinophilic leukemia - chronic form

Chronic myeloid leukemia

Chronic myelomonocytic leukemia

JAK gene amplification

Chronic myeloproliferative diseases, myelodysplastic diseases, acute myeloid leukemia

Determined by duplications, trisomias or unbalanced translocations

Present in chronic myeloproliferative diseases, myelodysplastic diseases associated to JAK2V617F mutation, which sometimes precedes

Probable secondary to cytotoxic therapy

Associated to myeloid metaplasia with myelofibrosis, the missing piece

being also implicated in chronic lymphocytic leukemia

100

95

50-70

50-60

1-5

5

1
Rare

Rare

Associated to

JAK2V617F mutation

Target gene not identified

Target gene not identified

Target gene not identified

${ }^{\mathrm{a} S e e}$ ref. 68.

Table IV. Protein molecular markers associated with myeloproliferative syndromes. ${ }^{\mathrm{a}}$

\begin{tabular}{ll}
\hline Protein & \multicolumn{1}{c}{ End effect } \\
\hline$B C L-X L$ & $\begin{array}{l}\text { Overexpressed in Polycythemia vera as a result of anti-apoptotic effect of JAK-STAT on } \\
\text { the erythroid cells } \\
\text { Increased expression in JAK2 positive myeloproliferative diseases, possible implicated in } \\
\text { erythroid differentiation }\end{array}$ \\
Increased ARN levels in Polycythemia vera, without an increase in protein levels \\
MPL & $\begin{array}{l}\text { Decreased protein expression on the cell surface and aberrant glycosylation in } \\
\text { the chronic myeloproliferative disease - unclear role in pathogenesis }\end{array}$ \\
FIP1L1-PDGFRA & Hypereosinophilic syndromes
\end{tabular}

a See ref. 68. FIP1L1, FH interacting protein 1-like 1; PDGFRA, platelet-derived growth-factor receptor $\alpha$ polypeptide; PDGFRB, plateletderived growth-factor receptor $\beta$ polypeptide; FGFR, fibroblast growth-factor receptor; BCL-XL, B-cell leukemia/lymphoma 2-like protein X long-transcript variant; NFE2, nuclear factor erythroid-derived 2; PRV1, Polycythemia rubra vera 1; and MPL, thrombopoietin receptor.

Janus Kinase 2 (JAK2) (6-10); ii) mutations of the gene encoding the thrombopoietin receptor (MPL) $(11,12)$; iii) mutations of the genes for the platelet derived growing factor ( $\alpha$-PDGFRA and less frequent $\beta$-PDGFRB), represented by deletions in chromosome 4 , leading to fusion gene FIP1L1-PDGFRA in hypereosinophilic syndrome (HES) (13); iv) mutations for receptor 1 of the fibroblastic growing factor (FGFR1) (14) or KIT gene mutations (15) encoding stem-cell factor receptor, which occur in systemic mastocytosis. 


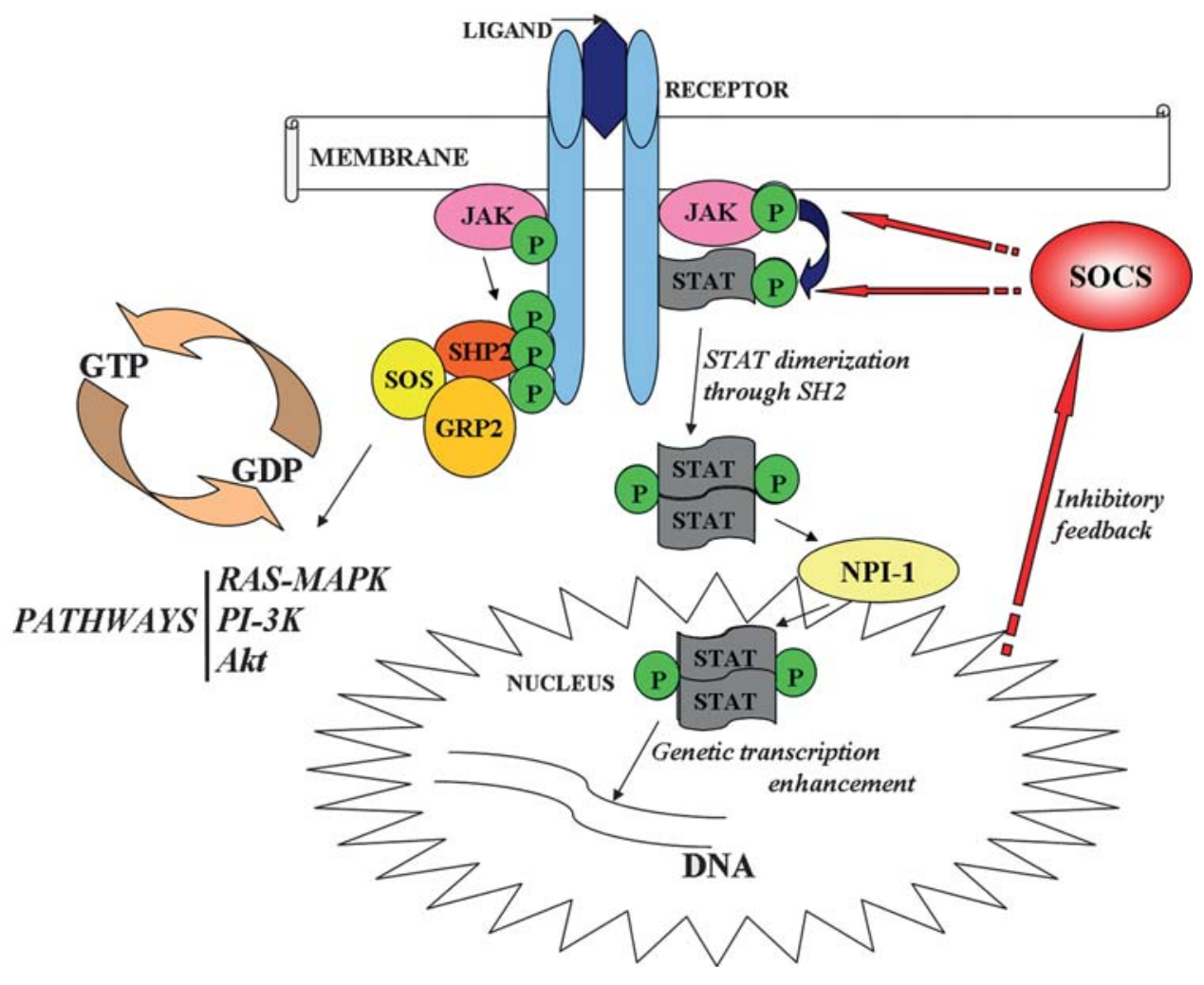

Figure 1. JAK-STAT signalling pathways and the JAK2V617F mutation. The figure depicts main signaling pathways including JAK-STAT signaling (modified after 16).

\section{The role of molecular markers in myeloproliferative} diseases

JAK2V617F allele. JAK 2 represents one of the 4 januskinases that play a role in intracellular signaling. The association of JAK2V617F mutation with myeloproliferative disorders: Polycythemia vera (PV), essential thrombocythemia (ET) and myelofibrosis with myeloid metaplasia (MMM) was first reported in $2005(8,9)$ and is considered by far the most common mutation in BCR-ABL negative CMPD. The etiology of the mutation is not known, but is was recently discovered that patients with a specific single nucleotide polymorphism (SNP) pattern at the JAK2 gene harbor a significantly increased risk to develop a JAK2 mutations and concomitant CMPD (Fig. 1).

JAK2V617F is generated by a somatic mutation of guanosine into usually thymidine in the JAK2 gene which results in the substitution of valine with phenylalanine at codon 617 . The mutation JAK2V617F activates ligand independent activation of downstream targets. As a consequence, transition from $\mathrm{G} 1$ phase to $\mathrm{S}$ phase of the cell cycle is accelerated. JAK2V617F induces BCL-XL and cyclin D2 protein expression. The JAK2 mutation also inhibits receptormediated apoptosis and leads to a decrease in MPL receptor expression (2). In cell lines, JAK2V617F stimulates cell growth independent of cytokines such as IL3 and erythropoietin, being associated with constitutive activation of JAK2, STAT5 and PI3K pathways.

Two molecules of each protein attach through the FERM domain to the intracellular part of a type 1 heterodimeric cytokine receptor, like the erythropoietin receptor, G-CSF receptor and MPL or of a type 1 or 2 heterodimeric cytokine receptor such as the receptor family for IL3 or INF- $\gamma$, starting a transphosphorylation process between the two components of the receptor. Then JAK2 phosphorylates the receptor's tyrosine residues, and initiates the process of recruiting the next molecules into the signaling chain: PI3K, RAS and STAT5. Each of these molecules is the starting point of some signaling cascades implicated into the regulation of proliferation, differentiation and apoptosis of the cells.

The JAK2V617F substitution is found in the homology domain $2(\mathrm{JH} 2)$ that plays a role in the physiological inhibition of the tyrosine-kinase activity that is maintained in homology domain 1 (JH1). The mutant protein JAK2V617F is constitutively phosphorylated and leads to activation of the signaling pathways STAT5, MAPK/ERK and Pi3K/AKT. Full activation is still partially dependent of a ligand interaction with the type 1 homodimeric cytokine receptor. This might explain why the effect of this mutation is seen mainly in erythroid, myeloid and megakaryocytic cell lines that predominantly express these types of receptors.

Several findings clearly indicate JAK2 in PV pathogenesis (2): i) $95 \%$ of PV patients show a V617 mutation, and the others might harbor other JAK2 mutations; ii) mice transplanted with mutant JAK2 hematopoietic cells develop a clinical picture and natural history similar to PV: low serum erythropoietin level, splenomegaly with extramedullar hematopoiesis, megakaryocytic hyperplasia with myelofibrosis and late onset of anemia (17). Thrombocytosis is constantly 


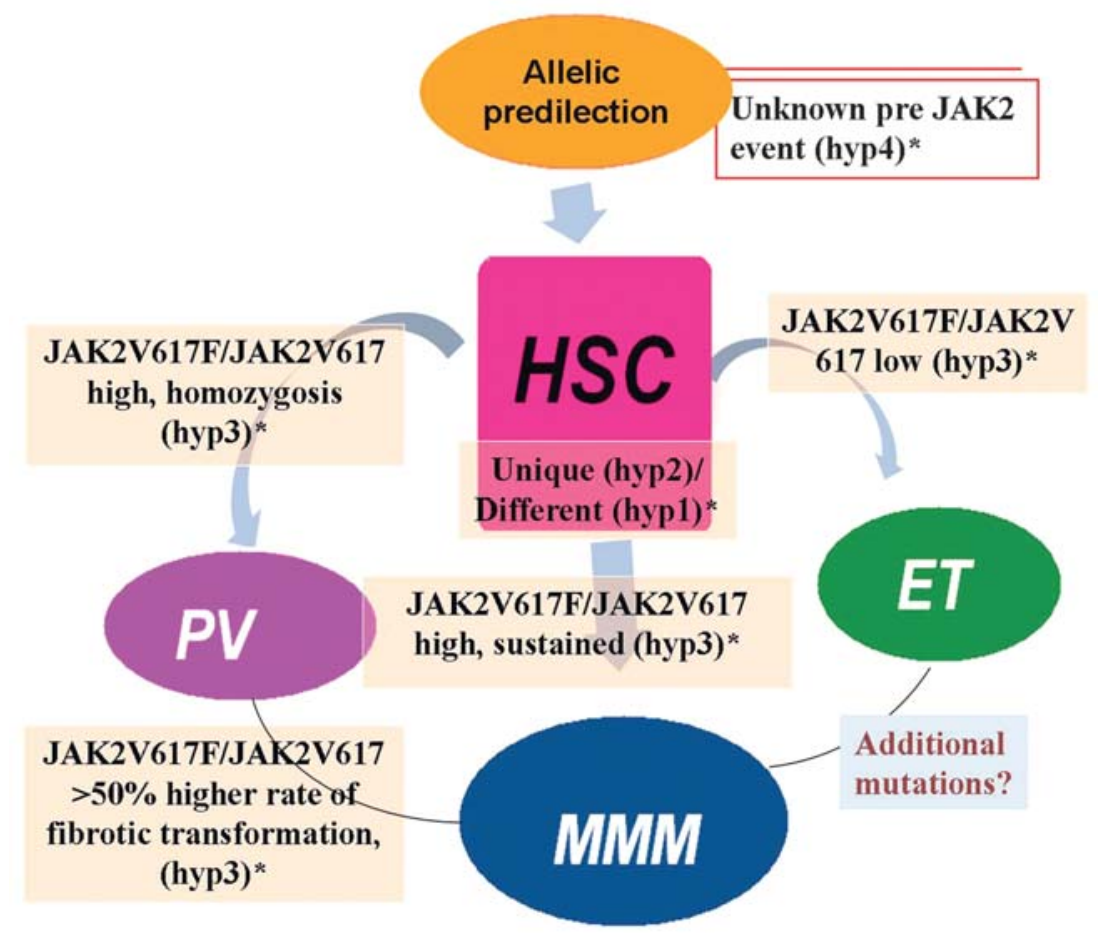

Figure 2. Oncopathogenic role of JAK2V617F mutation in MPN. Several hypotheses exist with regard to the pathogenetic mechanisms of JAK2 mutations. Four main hypotheses are graphically represented. The asterisks and numbers mention the different hypothesis are explained in the text (modified from 31 ).

absent in PV mice, but it is the main feature in MMM mice associated with MPLW515L mutation. The presence of this mutation explains two features of PV patients - erythropoietin hypersensitivity and terminal amplification of the erythroid lineage.

The JAK2V617F mutation is also detected in other myeloproliferative disorders such as ET in $40-60 \%$ of cases and MMM in $50 \%$ of cases. There is a particular common phenotype of JAK2 positive mutant MPNs. As an example, JAK2V617F positive ET shares some common features with PV: i) higher level of hemoglobin and neutrophils; ii) erythropoiesis and granulopoiesis are more active in the bone marrow; iii) higher incidence of venous thrombosis and polycythemic transformation; iv) erythropoietin and serum ferritin levels lower than in JAK2V617F negative ET patients.

The JAK2 mutation was also identified in $20-25 \%$ of atypical MPN, in a few rare MPN such as HES, chronic neutrophilic leukemia, chronic myelomonocytic leukaemia (CMML); and in myelodysplastic disorders (MDS) or acute leukemia cellular lineages [for example, cellular lineage of humane erythroleukemia (HEL)], and also in few cases of acute myeloid leukemia (AML) (18). In patients with AML that harbor a JAK2 mutation it is possible that the mutation indicates detection of a transformed disease that initially presented as MPN (but was not diagnosed). Alternatively, there might also be cases where JAK2 mutations contribute to an initial disease manifestation. Interestingly, some MPN patients that progress to AML do not show JAK2 mutations in the leukemic blasts. In these cases secondary AML might have developed due to increased susceptibility of the bone marrow or as a consequence of prolonged cytoreductive therapy (e.g., by hydroxyurea). Kremer et al (19) described the JAK2V617F mutation in borderline CMPD/MDS, but could not find it in MDS with fibrosis.

Remacha et al (20) identified RARS-T entity (refractory anemia with ringed sideroblasts with thrombocytosis) to have common features with CMPD/MDS: hyper cellular bone marrow with important megakaryocytic hyperplasia and giant forms, with a tendency to evolve into fibrosis. This entity associates JAK2 mutation and it is thought to correlate MDStype erythropoiesis from RARS subtype, with ET-type myeloproliferative elements.

At present, there are four main hypotheses regarding the involvement of JAK2V617F mutation in the pathogenesis of chronic myeloid neoplasms (21) (Fig. 2): one explanation for the occurrence of different phenotypes as a result of the same oncogenic event is the occurrence of the initial oncogenic event in different hematopoietic progenitor cells. The specific phenotype is therefore a consequence of the properties of the mutated self renewing cell to differentiate into platelets for ET or into erythrocytes for PV. The relevance of this hypothesis is uncertain due to recent discoveries which report other differences in the HSC compartment of PV and MMM patients: JAK2 wild-type for PV and JAK2V617F MMM. These results are obtained in a NOD/SCID mouse model (22).

Another possible theory suggests that the mutation occurs in the same HSC compartment of each patient. The phenotype then differs due to genetic specificity and heterogeneity of each case. This conclusion was first based on experiments in mice in which the same JAK2V617F transduced cells were transplanted in different species of mutation- 
specific research mice generating different phenotypes $(17,23,24)$. Recent genotyping of single nucleotide polymorphism (SNP) in the genes for JAK2, G-CSR and receptors for MPL and Epo of patients with MPN showed that certain SNPs of JAK2 and EpoR were particularly associated with PV or ET suggesting that phenotypic variation is the result of the genetic diversity (25).

The 'dosage model' proposes the mechanism of the phenotype diversity as a consequence of the variability in the number of the JAK2 allele copies from one patient to another. It regards all the JAK2V617F MPN as a single disease with different evolution stages due to the JAK2 kinase activity level. A low level of enzyme will generate ET, a high level will cause PV and the long persistence of an increase JAK2 kinase activity might finally induce myelofibrosis (26).

This hypothesis was first generated by Moliterno et al (27) in 1998. They reported a $67 \%$ level of JAK2V617F in neutrophils of PV patients (range 35-100\%), compared to $47 \%$ level in neutrophils of ET patients (range 10-63\%, p $\leq 0.001$ ). Vainchenker and his team $(2,26)$ raised the hypothesis that the level of JAK2V617F-kinase activity is crucial in differential diagnosis of different forms of MPN. This hypothesis is also supported by the diagnostic finding that JAK2V617F in PV is often homozygous whereas ET rather presents with heterozygous mutations. In 2008, Tiedt and collaborators showed a strong link between the JAK2V617F/JAK2 wildtype ratio and the MPN phenotype (28)

'The pre JAK2 mutation-event hypothesis' suggests that the different phenotypes would be the result of different genetic events that occur in HSC before the burst of JAK2 mutation. The most striking evidence is the presence of different JAK2V617F MPN in the same families and also the observation that the JAK2 mutation is absent in these patients $\mathrm{B}$ and $\mathrm{T}$ lymphocytes, advocating that this mutation is an acquired event and that an unknown germ cell factor predisposes to multiple MPN $(29,30)$.

From a clinical point of view, a general concern regarding the MPN is the risk of developing arterial and venous thrombosis. A study on 806 patients diagnosed with ET reported an association between the occurrence of JAK2V617F mutation and the venous thrombosis (32). Also, an increase in the prevalence of thrombotic events was observed in JAK2V167F MMM patients (33). Multiple studies, identified older age (32-34) higher haematocrit level $(32,34,35)$ and higher white blood cells number $(32,34)$ in relation with JAK2V617 mutation but not obvious risk factors for arterial and venous thrombosis collectively (34). Nevertheless homozygous mutations correlated with a higher risk of fibrosis and a higher level of hemoglobin (36).

Studying a group of 116 patients diagnosed with PV, Vannucchi et al (37) reported correlations between clinical parameters and homo/heterozygous status. A significant direct association was found between intracellular JAK2V617F and haematocrit, WBC, LDH, alkaline phosphatase levels, and an indirect relation with medium erythrocyte volume and platelet number.

Patients with a high fraction of mutant JAK2 allele/wildtype JAK2 allele (homozygous) at diagnosis are associated in a higher percentage with splenomegaly and pruritus and they need chemotherapy more often than other patients. A multivariate analysis including age, leucocytosis, haematocrit, platelet number and therapy options established the above mentioned fraction as an independent risk factor for major vascular events ( $\mathrm{p}=0.027)(37)$.

Another study confirmed these findings (38) in a group of 1306 patients with MPN and established an incidence of thrombosis at diagnosis of 12, 21 and $15 \%$, in wild-type PV, heterozygous and homozygous for JAK2V617F mutation. For ET patients, the percentage of patients with thrombosis at diagnosis were 11, 22 and 50\%, and for MMM patients 1 , 7 and $3 \%$. The relative risk for thrombosis during disease evolution (adjusted to age, sex and previous thrombosis) was significantly higher in ET patients and in MMM patients.

A recent analysis observed a statistically pertinent correlation between the allele burden $(>25 \%)$ and the presence of only arterial thrombosis in ET patients (39). The JAK2V617F mutation was also identified as an independent risk factor for pregnancy complications in ET women (40).

Baxter et al identified an association of JAK2 in 59\% of patients with Budd-Chiari syndrome (BCS) (7). Also, Smalberg et al (41) found a $41 \%$ prevalence of this mutation in BCS patients, on a group of 40 patients with primary nonmalignant BCS. As a consequence, all patients with BuddChiari syndrome that lack a clear reason for thrombosis should be analyzed for the presence of an occult myeloproliferative syndrome (42).

Regarding the impact on survival, the results are controversial; there are studies from the Mayo Clinic which did not detect any role of the mutant JAK2 V617F status as an independent prognostic factor for the survival in ET (34) or MMM (33). A multicentre European study (43) reported inferior survival in mutation positive MMM and ET patients, possibly related to the higher risk of leukemic transformation. Also, the JAK2V617F mutation in ET patients was associated with the evolution towards PV (34).

The JAK2 mutation also affects the response to treatment. Among ET patients, those with V617F mutation are more sensitive to hydroxycarbamidum, but not to anagrelide, compared to patients without mutation. Importantly, treatment response could be evaluated directly by quantitation of JAK2-positive peripheral blood cells. This is especially important for patients undergoing allogenic hematopoietic stem cell transplantation. The development of specific JAK2 inhibitors might allow disease monitoring similar to BCRABL positive CML (44).

In 2008 the 4th edition of the WHO classification of Haematopoietic Tumors established the presence of the JAK2V617F mutation as a clonality marker and a major criteria for diagnosis in PV, ET and MMM.

$M p l$ - thrombopoietin receptor. Thrombopoietin represents an important regulator of megakaryopoiesis and thrombopoiesis through the thrombopoietin receptor c-Mpl, encoded by the proto-oncogene c-Mpl. This receptor is expressed on $\mathrm{CD} 4^{+}$hematopoietic progenitors, megakaryocytes and platelets. Mutations in $\mathrm{Mpl}$ and in the erythropoietin receptor were initially identified in familial erythrocytosis and thrombocytosis.

Pikman and his group discovered that Mpl mutations have an activating effect on intracellular JAK2/STAT pathway, 
similar to JAK2V617F mutation. Thus, MPL ${ }^{\text {w515L }}$ mutation, represented by the substitution of tryptophan with leucine at the junction of transmembrane domain with cytoplasmic domain of MPL leads to a cytokine-independent cell proliferation and constitutive phosphorilation of JAK2, STAT, AKT and ERK (11).

Transplanting Mpl mutant cells in mice induces a lethal MPN characterized by leucocytosis, severe myelofibrosis with megakaryocytic hyperplasia, and severe thrombocytosis, without erythrocytosis. In a group of 1182 patients with different hematological diseases, of which 579 patients were diagnosed with ET and MMM, MPL mutation was present in $5.5 \%$ of MMM patients and $1.2 \%$ of ET patients, but not in PV patients, MDS, AML or CMML patients. The association of mutant Mpl with mutant JAK2 was very rare and occurred only in 2 ET patients and 4 MMM patients (11).

On the cell surface, JAK 2 mutation leads to downregulation of $\mathrm{c}-\mathrm{Mpl}$ expression $(45,46)$ suggesting that the decrease of c-MPL expression in PV and MMM is a characteristic of these diseases, being also an element of differential diagnosis between PV and other types of erythrocytosis.

From the clinical and diagnostic point of view the MPLW515-positive ET patients versus the JAK2V617Fpositive ET patients have a higher number of platelets, lower measurement of hemoglobin (47) with higher erythropoietin level and reduced bone marrow general and erythroid cellularity and not erythroid colonies but megakaryocytic colonies growth (48). In addition the MPLW515-mutated MMM patients have also a more severe anemia with an increased transfusion support (49).

The thrombotic role of the $M P L$ mutation is not well established yet. It was noted as a significant risk factor for microvessel disturbances, probably due to constitutively active MPL platelets. This augmented the occurrence of arterial thromboses only in comparison to $M P L$ wild-type/ $J A K 2$ wild-type patients (47). In consequence, the MPL W515K/L mutation represents a clonality marker considered as major diagnosis criterion for the diagnosis of MMM and ET (WHO classification 2008).

$P R V 1$. PRV1 is a surface protein from the uPAR/Ly6/ CD59 receptor family (50). PRV1 mRNA is overexpressed in granulocytes of PV and ET patients (51-54). Depending on the detection technique, independent laboratories have reported PRV1 expression in up to $90 \%$ of PV patients (55).

In 2005, a prospective study on 88 patients with MPN from Mayo Clinic (56) has quantified PRV1 expression in neutrophils by RT-PCR. Their results indicated overexpression in $83 \%$ of PV patients, $21 \%$ of ET patients, $42 \%$ of MMM patients and $18 \%$ of pseudo-polycythemia patients, compared to $0 \%$ in healthy controls. It is important to mention that all the MMM patients with overexpression of PRV1 developed MMM secondary to PV. All of these points suggest that overexpression of PRV1 represents a characteristic of PV which is not affected by the fibrotic transformation of the bone marrow. The diagnostic relevance of PRV1 in the era of JAK2 mutation detection technology is currently still uncertain. It might help in patients with characteristic disease features that lack the defining JAK2V617F mutation.
The level of expression of PRV1 also correlates with the risk of thrombosis. Griesshammer et al (57) identified the group of PRV1 positive ET as a separate entity, characterized by common features with PV regarding the thrombotic events and the evolution to PV. The PRV1 positive ET types represent $40 \%$ of ET.

Goerttler et al (58) identified overexpression of PRV1 as an independent risk factor for thromboembolic complications, while the down-regulation of the cMPL expression was not associated with the same risk. In conclusion the WHO classification 2008 did not include the PRV1 as a diagnosis marker for PV.

PDGFRA, PDGFRB and FGRF1. Platelet derived growth factor receptors (PDGFRs) are transmembrane tyrosine kinase receptors that consist of an extracellular ligand-binding domain, a transmembrane domain and a cytoplasmic tyrosine kinase domain (59). They can bind at least five types of active PDGF heterodimers (PDGF-AA, PDGF-AB, PDGF$\mathrm{BB}, \mathrm{PDGF}-\mathrm{CC}$ and PDGF-DD). These factors are released mainly from platelet $\alpha$-granules and act as potent mitogenic and chemotactic signals for a variety of mesenchymal cells. Affected cells include fibroblasts, vascular smooth muscle cells, glomerular mesangial cells and brain grail cells.

There are two receptor isoforms [PDGF receptor- $\alpha$ (PDGFRA) and $-\beta$ (PDGFRB) (60)] which also dimerize upon binding of the PDGF heterodimer ligand. Three possible receptor combinations occur $(\alpha \alpha, \beta \beta$ and $\alpha \beta)$ that activate the tyrosine kinase. The intracellular signaling cascade initiated by these receptors include Ras/Mitogen-activated protein kinase (MAPK) and PI-3 kinase as well as phospholipase- $\gamma$ (PLC $\gamma$ ) pathways.

The 2008 WHO classification of the Hematopoietic Tumors contains a new category of MPNs associated with rearrangements of PDGFRA, PDGFRB and FRGF1 genes that generate an abnormal tyrosine kinase (1).

PDGFRA related disorders include chronic eosinophilic leukemia (CEL) with an involvement of the mast cell lineage and often the neutrophil lineage. PDGFRA rearrangements were also detected in some rare cases of acute myeloid leukemia and precursor T lymphoblastic lymphoma (T-LBL). The most frequent fusion gene implicated in PDGFRAassociated MPN is FIP1L1-PDGFRA. This mutation is characterized by the loss of around $800 \mathrm{~kb}$ DNA sequence at chromosome $4 \mathrm{q} 12$ and cannot be detected by conventional cytogenetics. The intra-chromosomal deletion leads to the aberrant chimeric fusion protein FIP1L1-PDGFRA.

This relatively rare (14-16\%) (61) form of a hypereosinophilic syndrome was also called the 'myeloproliferative variant of the hypereosinophilic syndrome'. In the absence of genetic evidence it can be suspected in a BCR-ABL negative MPN with splenomegaly and hematological features of CEL, high number of mast cells in the bone marrow, serum tryptase level $(>12 \mathrm{ng} / \mathrm{ml})$ and markedly raised $B_{12}$ vitamin level. From the clinical point of view, CEL presents with a more severe phenotype, with extended organ involvement (1). In these diseases, the heart, lung, gastrointestinal tract, skin and the nervous system (central and peripheral) are commonly affected, as a consequence of the cytokines release. Imatinib inhibits PDGFRA activity and frequently induces 
remissions in patients with eosinophilic leukemias. The FIP1L1-PDGFRA gene product is 100 times more sensitive to imatinib than BCR-ABL. Similar to CML management; imatinib resistance may develop as a result of the T674I mutation. This mutation is the equivalent of the T315I mutation in BCR-ABL positive disorders. Other very rarely observed PDGFRA rearrangements (KIF5B-PDGFRA, CDK5RAP2-PDGFRA, STRN-PDGFRA, ETV6-PDGFRA, BCR-PDGFRA) have also responded to imatinib therapy.

In the case of PDGFRB rearrangements, the most frequent clinical finding is chronic myelomonocytic leukaemia with eosinophilia, although aberrant proliferation of mast cells may be a consequence. Very rare cases of transformation in acute myeloid leukemia have been described.

The genetic location of PDGFRB rearrangements is at 5q31-311 chromosome. This anomaly often generates the ETV6-PDGFRB fusion gene $[t(5,12)]$ with clinical features of CMML accompanied by eosinophilia. There are also many exceptions which might mimic a clinical picture of CMML (RABEP1-PDGFRB, NDE1-PDGFRB), of CMML with eosinophilia (KIAA1509-PDGFRB, TP53PB1-PDGFRB, HIP1-PDGFRB, NIN-PDGFRB), of CEL (TPM3-PDGFRB, GIAP1-PDGFRB, WDR48-PDGFRB, GIT2-PDGFRB), chronic basophilic leukemia, atypical CML with eosinophilia, juvenile myelomonocytic leukemia, MPD or MPD/MDS with eosino-philia. Data on a rather small number of cases show that the poor survival of these patients $(<2$ years) can also be improved by imatinib (62).

FGFR-1 positive diseases often present as T-LBL with eosinophilia. There are also reported cases with this genetic rearrangements and CEL, precursor B lymphoblastic lymphoma/leukemia or AML. The genetic aberrations affect the pluripotent lymphoid-myeloid stem cell and are composed by a variety of translocations at $8 \mathrm{p} 11$ breakpoint. In all cases abnormal tyrosine kinase activity is observed for whom no TK inhibitor treatment has been firmly established (1).

c-KIT. This type III receptor tyrosine kinase is involved in a variety of developmental processes. It is the receptor of the stem cell factor (SCF). The gene encodes a five loop transmembrane immunoglobulin-like structure receptor for the stem cell growth factor (SCF), a member of the PFGF and CSF-1 receptor family (63). An intracellular kinase insert splits the catalytic domain into the ATP binding site and the phosphotransferase active site.

The c-kit receptor (CD117) is expressed on mast cells, hematopoietic stem cells, melanocytes and germ cell lineages where it is involved in mediating critical signals for the growth and maturation of these cells (64) especially via the PI3K-Akt pathway (65). Normal kit signaling contributes to hematopoiesis, mast cell development and function, gametogenesis and melanogenesis. Mutations associated with loss of function generate an autosomal dominant condition characterized by depigmented patches of skin and hair named piebaldism. c-kit is altered in several malignancies. Overexpression and mutations of c-kit are found in myeloproliferative disorders, AML, especially core binding factor acute myeloid leukemia and systemic mastocytosis (SM). In addition, c-kit alterations occur in germ cell tumors, gastrointestinal stromal tumors, sino-nasal $\mathrm{T}$ cell lymphomas and myelodysplastic syndromes (66). In patients with mastocytosis, the tyrosine kinase domain of the SCF receptor encoded by the KIT proto-oncogene frequently acquires a somatic point mutation.

The most common site involved is the codon 816 with a change of the amino acid valine to aspartic acid. This induces a self-activated tyrosine kinase which is resistant to classical tyrosine kinase inhibitors such as imatinib (D816V mutation). Sensitive PCR-based laboratory methods identified D816V in $\sim 95 \%$ adults with systemic mastocytosis and one third of the children with cutaneous mastocytosis. Several clinical trials are underway that specifically target this mutation. The presence of an activating point mutation at codon 816 of Kit in bone marrow, peripheral blood cells or extracutaneous organs is considered a minor criterion in the newly revised WHO classification of hematopoietic neoplasms.

\section{Conclusions}

The dramatic therapeutic progress achieved due to inhibition of BCR-ABL in CML has initiated a novel era of genetic and molecular discoveries in hematological disorders. Currently, specific mutations are found in many patients with suspected myeloproliferative disorders. This led to new diagnostic classification. The rapid invention of specific inhibitors for the mutated kinases provides hope that specific therapies can be developed for BCR-ABL negative myeloproliferative syndromes. It can be anticipated that further identification of novel mutations will define novel and so far unrecognized disease entities.

\section{References}

1. Swerdlow SH, Campo E, Lee Harris NL, Jaffe ES, Pileri SA, Stein H, Thiele J and Vardiman JW: WHO classification of tumors of haematopoietic and lymphoid tissue. IARC Press, pp14-86, 2008.

2. Vainchenker W, Deholmmeau F and Villeval JL: Molecular pathogenesis of the myeloproliferative diseases. Education program for the 12t Congress of the European Hematology Association, Vienna, Austria, June 7-10, 2007. Haematologica 1: 239-246, 2007.

3. Nowell PC and Hungerford DA: Chromosome studies on normal and leukemic human leukocytes. J Natl Cancer Inst 25: 85-109, 1960.

4. Rowley JD: Letter: a new consistent chromosomal abnormality in chronic myelogenous leukaemia identified by quinacrine fluorescence and Giemsa staining. Nature 243: 290-293, 1973.

5. Bartram CR, De Klein A, Hagemeijer A, et al: Translocation of $\mathrm{c}$-abl oncogene with the presence of a Philadelphia chromosome in chronic myelocytic leukemia. Nature 306: 277-280, 1983.

6. James C, Ugo V, Le Couédic JP, Staerk J, Delhommeau F, Lacout C, Garçon L, Raslova H, Berger R, Bennaceur-Griscelli A, Villeval JL, Constantinescu SN, Casadevall N and Vainchenker W: A unique clonal JAK2 mutation leading to constitutive signalling causes polycythaemia vera. Nature 434: 1144-1148, 2005

7. Baxter EJ, Scott LM, Campbell PJ, East C, Fourouclas N, Swanton S, Vassiliou GS, Bench AJ, Boyd EM, Curtin N, Scott MA, Erber WN and Green AR: Acquired mutation of the tyrosine kinase JAK2 in human myeloproliferative disorders. Lancet 365: 1054-1061, 2005.

8. Levine RL, Wadleigh M, Cools J, et al: Activating mutation in the tyrosine kinase JAK2 in polycythemia vera, essential thrombocythemia and myeloid metaplasia with myelofibrosis. Cancer Cell 7: 387-397, 2005.

9. Kralovics R, Passamonti F, Buser AS, et al: A gain of function mutation in JAK2 is frequently found in patients with myeloproliferative disorders. N Engl J Med 352: 1779-1790, 2005. 
10. Zhao R, Xing S, Li Z, Fu X, Li Q, Krantz SB and Zhao ZJ: Identification of an acquired JAK 2 mutation in Polycythemia vera. J Biol Chem 280: 22788-22792, 2005

11. Pikman Y, Lee BH, Mercher T, McDowell E, Ebert BL, Gozo M, Cuker A, Wernig G, Moore S, Galinsky I, DeAngelo DJ, Clark JJ, Lee SJ, Golub TR, Wadleigh M, Gilliland DG and Levine RL: MPLW515L is a novel somatic mutation in myelofibrosis with myeloid metaplasia. PloS Med 3: E270, 2006.

12. Pardanani AD, Levine RL Lasho T, Pikman Y, Mesa RA Wadleigh M, Steensma DP, Elliott, MA, Wolanskyj AP, Hogan WJ, McClure RF, Litzow MR, Gilliland DG and Tefferi A: MPL515mutations in myeloproliferative and other myeloid disorders: a study of 1182 patients. Blood 108: 3472-3476, 2006.

13. Cools J, DeAngelo DJ, Gotlib J, et al: A tyrosine kinase created by fusion of the PDGFRA and FIP1L1 genes as therapeutic target of imatinibin in idiopathic hypereosinophillic syndrome. N Engl J Med 348: 1201-1214, 2003.

14. Reiter A, Sohal J, Kulkarni S, Chase A, Macdonald DH, Aguiar RC, Gonçalves C, Hernandez JM, Jennings BA, Goldman JM and Cross NC: Consistent fusion of ZNF198 to the fibroblast growth factor receptor-1 in the $t(8 ; 13)(\mathrm{p} 11 ; \mathrm{q} 12)$ myeloproliferative syndrome. Blood 92: 1735-1742, 1998.

15. Nagata H, Worobec AS, Oh CK, Chowdhury BA, Tannenbaum S, Suzuki Y and Metcalfe DD: Identification of a point mutation in the catalytic domain of the protooncogene c-kit in peripheral blood mononuclear cells of patients who have mastocytosis with an associated hematologic disorder. Proc Natl Acad Sci USA 92: 10560-10564, 1995

16. McLornan D, Percy M and McMullin MF: JAK2 V617F: a single mutation in the myeloproliferative group of disorders. Ulster Med J 75: 112-119, 2006.

17. Wernig G, Mercher T, Okabe R, Levine RL, Lee BH and Gilliland DG: Expression of JAK2V617F causes a polycythemia vera-like disease with associated myelofibrosis in a murine bone marrow transplant model. Blood 107: 4274-4281, 2006.

18. James C, Ugo V, Casadevall N, Constantinescu SN and Vainchenker W: A JAK2 mutation in myeloproliferative disorders: pathogenesis and therapeutic and scientific prospects. Trends Mol Med 11: 546-554, 2005.

19. Kremer M, Horn T, Dechow T, Tzankov A, QuintanillaMartinez L and Fend F: The JAK2 V617F mutation occurs frequently in myelodysplastic/myeloproliferative diseases, but is absent in true myelodysplastic syndromes with fibrosis. Leukemia 20: 1315-1316, 2006.

20. Remacha AF, Nomdedéu JF, Puget G, Estivill C, Sarda MP Canals $\mathrm{C}$ and Aventin A: Occurrence of the JAK V617F mutation in the WHO provisional entity: myelodysplastic/myeloproliferative disease, unclassifiable-refractory anemia with ringed sideroblasts associated with marked thrombocytosis. Haematologica 91: 719-720, 2006

21. James C: The JAK2V617F mutation in Polycythemia vera and other myeloproliferative disorders: one mutation for three diseases? Am Soc Hematol Educ Program Book, pp69-75, 2008.

22. James C, Mazurier F, Dupont S, Chaligne R, Lamrissi-Garcia I, Tulliez M, Lippert E, Mahon FX, Pasquet JM, Etienne G, Delhommeau F, Giraudier S, Vainchenker W and De Verneuil H: The hematopoietic stem cell compartment of JAK2V617F-positive myeloproliferative disorders is a reflection of the disease heterogeneity. Blood 112: 2429-2438, 2008.

23. Lacout C, Pisani DF, Tulliez M, Gachelin FM, Vainchenker W and Villeval JL: JAK2V617F expression in murine hematopoietic cells leads to MPD mimicking human PV with secondary myelofibrosis. Blood 108: 1652-1660, 2006

24. Zaleskas VM, Krause DS, Lazarides K, Patel N, Hu Y, Li S and van Etten RA: Molecular pathogenesis and therapy of polycythemia induced in mice by JAK2 V617F. PLoS ONE 1: E18, 2006.

25. Pardanani A, Friedly B1, Lasho TL, Gilliland DG and Tefferi A: Host genetic variations contributes to phenotypic diversity in myeloproliferative disorders. Blood 111: 2785-2789, 2008

26. Villeval JL, James C, Pisani DF, Casadevall N and Vainchenker W: New insights into the pathogenesis of JAK2 V617F-positive myeloproliferative disorders and consequence for the management of the patients. Semin Thromb Hemost 32: 341-351, 2006

27. Moliterno AR, Hankins WD and Spivak JL: Impaired expression of the thrombopoietin receptor by platelets from patients with Polycythemia vera. N Engl J Med 338: 572-580, 1998
28. Tiedt R, Hao-Shen H, Sobas MA, Looser R, Dirnhofer S, Schwaller J and Skoda RC: Ratio of mutant JAK2-V617F to wild-type Jak2 determines the MPD phenotypes in transgenic mice. Blood 111: 3931-3940, 2008.

29. Kralovics R, Stockton D and Prchal J: Clonal hematopoiesis in familial Polycythemia vera suggests that involvement of multiple mutational events in the early pathogenesis of the disease. Blood 102: 3793-3796, 2003

30. Bellanné-Chantelot C, Chaumarel I, Labopin M, Bellanger F Barbu V, De Toma C, Delhommeau F, Casadevall N, Vainchenker W, Thomas G and Najman A: Genetic and clinical implications of the Val617Phe JAK2 mutation in 72 families with myeloproliferative disorders. Blood 108: 346-352, 2006.

31. Levine R and Wernig G: Role of JAK-STAT signaling in the pathogenesis of myeloproliferative disorders. Am Soc Hematol Educ Book, pp233-239, 2006.

32. Campbell PJ, Scott LM, Buck G, et al: Definition of subtypes of essential thrombocythemia and relation to Polycythemia vera based on JAK2V617F mutation status: a prospective study. Lancet 366: 1945-1953, 2005

33. Tefferi A, Lasho TL, Schwager SM, Steensma DP, Mesa RA, Li CY, Wadleigh M and Gary Gilliland D: The JAK2(V617F) tyrosine kinase mutation in myelofibrosis with myeloid metaplasia: lineage specificity and clinical correlates. Br J Hematol 131: 320-328, 2005.

34. Wolanskyj AP, Lasho TL, Schwager SM, McClure RF, Wadleigh M, Lee SJ, Gilliland DG and Tefferi A: JAK2 mutation in essential thrombocythaemia: clinical associations and longterm prognostic relevance. Br J Hematol 131: 208-213, 2005.

35. Antonioli E, Guglielmelli P, Pancrazzi A, et al: Clinical implications of the JAK2 V617F mutation in essential thrombocythemia. Leukemia 19: 1847-1849, 2005.

36. Tefferi A, Lasho TL, Schwager SM, Strand JS, Elliott M, Mesa R, Li CY, Wadleigh M, Lee SJ and Gilliland DG: The clinical phenotype of wild-type, heterozygous and homozygous JAK2V617F in polycythemia vera. Cancer 106: 631-635, 2006.

37. Vannucchi AM, Antonioli E, Guglielmeli P, et al: Influence of the JAK2V617F mutational load at diagnosis on major clinical aspects in patients with Polycythemia vera. Am Soc Hematol Annu Meet Abstr 108: 5, 2006.

38. Vannucchi AM, Barosi G, Rimbaldi A, et al: Clinical significance of JAK2V617F homozygosity in the chronic myeloproliferative disorders. A study of 1306 patients. Am Soc Hematol Annu Meet Abstr 108: 664, 2006.

39. Antonioli E, Guglielmelli P, Poli G, Bogani C, Pancrazzi A, Longo G, Ponziani V, Tozzi L, Pieri L, Santini V, Bosi A and Vannucchi AM; Myeloproliferative Disorders Research Consortium (MPD-RC): Influence of JAK2V617F allele burden on phenotype in essential thrombocythemia. Haematologica 93: 41-48, 2008

40. Passamonti F, Randi ML, Rumi E, et al: Increased risk of pregnancy complications in patients with essential thrombocythemia carrying the JAK2(V617F) mutation. Blood 110: 485-489, 2007.

41. Smalberg JH, Murad SD, Braakman E, Valk PJ, Janssen HLA and Leebeek F WG: Myeloproliferative disease in the pathogenesis and survival of Budd-Chiari syndrome. Haematologica 91: 1712-1713, 2006

42. Patel RK, Lea NC, Heneghan MA, et al: Prevalence of the activating JAK2 tyrosine kinase mutation V617F in the BuddChiari syndrome. Gastroenterology 130: 2131-2138, 2006.

43. Campbell PJ, Griesshammer M, Dohner K, et al: V617F mutation in JAK2 is associated with poorer survival in idiopathic myelofibrosis. Blood 107: 2029-2100, 2006.

44. Tefferi A: JAK2 mutations in polycythemia vera, molecular mechanism and clinical applications. N Engl J Med 356: 444-445, 2007.

45. Horikawa Y, Matsumura I, Hashimoto K, Shiraga M, Kosugi S Tadokoro S, Kato T, Miyazaki H, Tomiyama Y, Kurata Y, Matsuzawa Y and Kanakura Y: Markedly reduced expression of platelet c-mpl receptor in essential thrombocythemia. Blood 90: 4031-4038, 1997

46. Tefferi A, Yoon SY and Li CY: Immunohistochemical staining for megakaryocyte c-mpl may complement morphologic distinction between polycythemia vera and secondary erythrocytosis. Blood 96: 771-772, 2000.

47. Vannucchi AM, Antonioli E, Guglielmelli P, et al: Characteristics and clinical correlates of MPL $515 \mathrm{~W}>\mathrm{L} / \mathrm{K}$ mutation in essential thrombocythemia. Blood 112: 844-847, 2008. 
48. Beer PA, Campbell PJ, Scott LM, et al: MPL mutations in myeloproliferative disorders: analysis of the PT-1 cohort. Blood 112: 141-149, 2008.

49. Guglielmelli P, Pancrazzi A, Bergamaschi G, et al: Anaemia characterises patients with myelofibrosis harbouring Mpl mutation. Br J Haematol 137: 244-247, 2007.

50. Temerinac S, Klippel S, Strunck E, et al: Cloning of PRV-1, a novel member of the uPAR receptor superfamily, which is overexpressed in polycythemia rubra vera. Blood 95: 2569-2576, 2000.

51. Klippel S, Strunck E, Temerinac S, et al: Quantification of PRV-1 mRNA distinguishes polycythemia vera from secondary erythrocytosis. Blood 102: 3569-3574, 2003.

52. Klippel S and Pahl HL: Molecular markers for the diagnosis of Philadelphia chromosome negative myeloproliferative disorders. Pathol Biol 52: 267-274, 2004.

53. Cilloni D, Carturan S, Gottardi E, et al: Usefulness of the quantitative assessment of PRV-1 gene expression for the diagnosis of polycythemia vera and essential thrombocythemia patients (letter). Blood 103: 2428, 2004.

54. Florensa L, Besses C, Zamora L, et al: Endogenous erythroid and megakaryocytic circulating progenitors, HUMARA clonality assay, and PRV-1 expression are useful tools for diagnosis of polycythemia vera and essential thrombocythemia. Blood 103: 2427-2428, 2004.

55. Liu E, Jelinek J, Pastore YD, Guan Y, Prchal JF and Prchal JT: Discrimination of polycythemias and thrombocytoses by novel, simple, accurate clonality assays and comparison with PRV-1 expression and BFU-E response to erythropoietin. Blood 101: 3294-3301, 2003.

56. Tefferi A, Lasho TL, Wolanskyj AP and Mesa RA: Neutrophil PRV-1 expression across the chronic myeloproliferative disorders and in secondary or spurious polycythemia. Blood 103: 3547-3548, 2004.

57. Griesshammer M, Klippel S, Strunck E, Temerinac S, Mohr U, Heimpel $\mathrm{H}$ and Pahl HL: PRV-1 mRNA expression discriminates two types of essential thrombocythemia. Ann Hematol 83: 364-370, 2004.
58. Goerttler PS, Steimle C, März E, Johansson PL, Andreasson B, Griesshammer M, Gisslinger H, Heimpel H and Pahl HL: The JAK2V617F mutation, PRV-1 overexpression and EEC formation define a similar cohort of MPD patients. Blood 106: 2862-2864, 2005.

59. Alvarez RH, Kantarjian HM and Cortes JE: Biology of plateletderived growth factor and its involvement in disease. Mayo Clin Proc 81: 1241-1257, 2006.

60. http://atlasgeneticsoncology.org/Genes/PDGFRBID21ch5q32. html, consulted 16.05.2009.

61. Yamada Y, Rothenberg ME, Lee AW, Akei HS, Brandt EB, Williams DA and Cancelas JA: The FIP1L1-PDGFRA fusion gene cooperates with IL-5 to induce murine hypereosinophilic syndrome (HES)/chronic eosinophilic leukemia (CEL)-like disease. Blood 107: 4071-4079, 2006.

62. David M, Cross NC, Burgstaller S, et al: Durable responses to imatinib in patients with PDGFRB fusion gene-positive and BCR-ABL-negative chronic myeloproliferative disorders. Blood 109: 61-64, 2007

63. Yarden Y, Kuang WJ, Yang-Feng T, Coussens L, Munemitsu S, Dull TJ, Chen E, Schlessinger J, Francke U and Ullrich A: Human protooncogene c-kit: a new cell surface receptor tyrosine kinase for an unidentified ligand. EMBO J 6: 3341-3351, 1987.

64. Nagata H, Worobec AS, Semere T and Metcalfe DD: Elevated expression of the proto-oncogene c-kit in patients with mastocytosis. Leukemia 12: 175-181, 1998.

65. Lim KH, Pardanani A and Tefferi A: KIT and mastocytosis. Acta Haematol 119: 194-198, 2008.

66. Siitonen T, Savolainen ER and Koistinen P: Expression of the c-kit proto-oncogene in myeloproliferative disorders and myelodysplastic syndromes. Leukemia 8: 631-637, 1994.

67. Tefferi A: Classification, diagnosis and management of myeloproliferative disorders in the JAK2V617F era. Hematology Am Soc Hematol Educ Book, pp240-245, 2006.

68. Campbell P and Green AR: The myeloproliferative disorders. N Engl J Med 355: 2452-2466, 2006. 